PROCEEDINGS OF THE

AMERICAN MATHEMATICAL SOCIETY

Volume 133, Number 11, Pages 3171-3177

S 0002-9939(05)07855-X

Article electronically published on May 4, 2005

\title{
IRREDUCIBLE POLYNOMIALS WHICH ARE LOCALLY REDUCIBLE EVERYWHERE
}

\author{
ROBERT GURALNICK, MURRAY M. SCHACHER, AND JACK SONN
}

(Communicated by Martin Lorenz)

\begin{abstract}
For any positive integer $n$, there exist polynomials $f(x) \in \mathbb{Z}[x]$ of degree $n$ which are irreducible over $\mathbb{Q}$ and reducible over $\mathbb{Q}_{p}$ for all primes $p$ if and only if $n$ is composite. In fact, this result holds over arbitrary global fields.
\end{abstract}

\section{INTRODUCTION}

Hilbert gave examples of irreducible polynomials $f(x) \in \mathbb{Z}[x]$ of degree 4 which are reducible mod $p$ for all primes $p$, namely $x^{4}+2 a x^{2}+b^{2}$. Note that this polynomial is irreducible over $\mathbb{Q}(a, b)$, hence (by Hilbert's irreducibility theorem) is irreducible over $\mathbb{Q}$ for infinitely many specializations of $a$ and $b$ into $\mathbb{Q}$. The underlying reason for this phenomenon from the Galois-theoretic point of view is that the Galois group of $x^{4}+2 a x^{2}+b^{2}$ over $\mathbb{Q}(a, b)$ is Klein's four group. Therefore for any $p$ not dividing the discriminant of $f$, the decomposition group is a cyclic group of order at most 2 , so $f$ is reducible $\bmod p$. (Note that for $p$ dividing the discriminant of $f, f$ is reducible $\bmod p$ as well.) The phenomenon is thus forced by the structure of the Galois group. This also explains why there can be no such examples of polynomials of prime degree. Indeed, suppose $f(x) \in \mathbb{Z}[x]$ has prime degree $\ell$ and is irreducible in $\mathbb{Z}[x]$. Then its Galois group has an element of order $\ell$, so by Chebotarev's density theorem there exists $p$ such that the splitting field of $f$ over $\mathbb{F}_{p}$ has Galois group $C_{\ell}$, the cyclic group of order $\ell$; hence $f$ must be irreducible over $\mathbb{F}_{p}$. We will give a proof that the degree of $f$ being prime is the only obstacle, namely that for any composite $n$, there exist irreducible $f(x) \in \mathbb{Z}[x]$ of degree $n$ which are reducible $\bmod p$ for all $p$. Brandl [2] has proved the same result by similar methods. We give a short proof of a generalization of this.

In fact, there is an irreducible $f(t, x) \in \mathbb{Z}[t, x]$ of degree $n$ such that $f\left(t_{0}, x\right)$ is reducible $\bmod p$ for all specializations $t=t_{0}$ in $\mathbb{Z}$ and all $p$. We will also prove the more delicate result that for any composite $n$, there exist irreducible $f(x) \in \mathbb{Q}[x]$ of degree $n$ which are reducible over $\mathbb{Q}_{p}$ for all $p$, and that this result generalizes to arbitrary global fields. Note that Hilbert's example does not satisfy this last condition for all $a, b$, e.g. $x^{4}+1$ is irreducible over $\mathbb{Q}_{2}$.

Received by the editors April 3, 2004 and, in revised form, June 17, 2004.

2000 Mathematics Subject Classification. Primary 11R52, 11S25, 12F05, 12G05, 16K50.

The first author was partially supported by NSF Grant DMS 0140578. The research of the third author was supported by Technion V.P.R. Fund-S. and N. Grand Research Fund. 
It is worthwhile pointing out here that a random polynomial $f(x) \in \mathbb{Z}$ of composite degree $n$ is not reducible $\bmod p$ for all $p$, as its Galois group over $\mathbb{Q}$ is $S_{n}[6]$, and since $S_{n}$ contains an $n$-cycle, Chebotarev's density theorem implies that there are infinitely many primes $p$ for which $f(x)$ is irreducible $\bmod p$.

\section{MOD $p$ REDUCIBILITY}

Let $f(x) \in \mathbb{Z}[x]$ be monic irreducible of degree $n$ with Galois group $G$ over $\mathbb{Q}$. As in the discussion above, we see that if $f(x)$ is irreducible mod $p$, then $p$ does not divide the discriminant of $f(x)$, and $G$ must contain an element of order $n$, since the decomposition group is cyclic of order $n$. Thus if $G$ has no element of order $n$, then $f$ must be reducible $\bmod p$ for all $p$. On the other hand, since $f$ is irreducible over $\mathbb{Z}, G$ has a subgroup $H$ of index $n$. More generally, if $K / \mathbb{Q}$ is a finite Galois extension with Galois group $G$, and if $G$ has a subgroup $H$ of index $n$ but no element of order $n$, then the fixed field, say $\mathbb{Q}(\theta)$, of $H$ has degree $n$ and the minimal polynomial $f$ of $\theta$ over $\mathbb{Q}$ has degree $n$ with splitting field $L \subseteq K$, Galois group $\bar{G}=G(L / \mathbb{Q}) \cong G / \operatorname{core}(H)$ where core $(H)$ is the intersection of the conjugates of $H$. Furthermore, $\bar{G}$ has a subgroup $\bar{H}$ of index $n$ but has no element of order $n$, so $f$ is reducible mod $p$ for every $p$. In summary:

Lemma 2.1. Let $G$ be a finite group and $n$ a positive integer such that

1) $G$ is realizable as a Galois group $G(K / \mathbb{Q})$,

2) $G$ has a subgroup of index $n$, but $G$ has no element of order $n$.

Then there is an irreducible polynomial $f(x) \in \mathbb{Z}[x]$ of degree $n$ which is reducible mod $p$ for all primes $p$ (with splitting field contained in $K$ ).

In connection with Hilbert's example, it follows from the preceding considerations that a polynomial of degree four has the property of being irreducible over $\mathbb{Z}$ and reducible mod $p$ for all $p$ if and only if its Galois group over $\mathbb{Q}$ is either Klein's four group $V_{4}$ or the alternating group $A_{4}$.

Theorem 2.2. For any composite positive integer $n$, there exist irreducible polynomials $f(x) \in \mathbb{Z}[x]$ of degree $n$ which are reducible $\bmod p$ for all primes $p$.

Proof. By the lemma, it suffices to find $G$ satisfying conditions 1) and 2).

Case 1. $n$ squarefree. Write $n=q m$ with $q$ prime, so $(m, q)=1$. Let $t$ be the order of $q \bmod m$ and set $G=C_{m} \ltimes V$ (semidirect product), where $V$ denotes the additive group of $\mathbb{F}_{q^{t}}$ with $C_{m}$ acting by multiplication by the $m$ th roots of unity $\mu_{m} \subset \mathbb{F}_{q^{t}}$, where we identify $C_{m}$ with $\mu_{m}$. The action of $C_{m}$ on $V$ is faithful and 0 is the only fixed point. Let $H$ be a hyperplane in $V$, i.e. $[V: H]=q$. Then $[G: H]=q m=n$. Furthermore, if $g \in G$ had order $n$, then $g^{m}(\in V)$ would have order $q$ and be fixed by $g$, hence by $C_{m}$, a contradiction.

We therefore have found for every squarefree composite $n$, a solvable group $G$ satisfying condition 2). By Shafarevich's theorem (in fact by an older theorem of Scholz), $G$ is realizable as a Galois group over $\mathbb{Q}$.

Case 2. $n$ not squarefree. Assume first that $n=q^{2}, q$ prime. Then $G_{1}:=C_{q} \times C_{q}$ satisfies 2) with $H$ the trivial group. For arbitrary $n=q^{2} m$, take $G:=G_{1} \times C_{m}$ which again has the trivial subgroup of index $n$ and no element of order $n$.

We now remark that since the groups $G$ appearing in the proof can be realized regularly over the rational function field $\mathbb{Q}(t)$ (see e.g. [4, p. 275]), we obtain irreducible polynomials $f(t, x) \in \mathbb{Z}[t, x]$ of degree $n$ which by Hilbert's irreducibility 
theorem have infinitely many specializations of $t$ into $\mathbb{Q}$ which are irreducible with Galois group $G$, hence reducible $\bmod p$ for all $p$.

\section{3. $p$-ADIC REDUCIBILITY}

We now wish to prove Theorem 2.2 with reducibility $\bmod p$ replaced by reducibility over $\mathbb{Q}_{p}$. The preceding construction actually yields irreducible polynomials $f(x) \in \mathbb{Z}[x]$ which are reducible over $\mathbb{Q}_{p}$ for all primes which are unramified in the splitting field of $f$, but may be irreducible over $\mathbb{Q}_{p}$ for ramified $p$. The proof will be similar but more delicate.

Let $f(x) \in \mathbb{Q}[x]$ be irreducible and let $p$ be a prime. Let $K$ be the splitting field of $f$ over $\mathbb{Q}, G=G(K / \mathbb{Q}), \mathfrak{p}$ a prime of $K$ over $p, D=D(\mathfrak{p})$ the decomposition group. Then $f$ is irreducible over $\mathbb{Q}_{p}$ if and only if $D(\mathfrak{p})$ acts transitively on the roots of $f$ in $K$. Let $H$ be the subgroup of $G$ fixing a root of $f$. The action of $G$ on the roots of $f$ is equivalent to its action by multiplication from the left on the left cosets of $H$ in $G$. Thus $f$ is irreducible over $\mathbb{Q}_{p}$ if and only if $D(\mathfrak{p})$ acts transitively on the left cosets of $H$ in $G$, i.e. the set product $D H$ is equal to $G$. Suppose $K / \mathbb{Q}$ were tamely ramified, so that all the decomposition groups were metacyclic. Then in order to insure that $f$ is reducible over all $\mathbb{Q}_{p}$, it would suffice that (the set product) $M H \neq G$ for every metacyclic subgroup $M$ of $G$. We therefore have the following lemma.

Lemma 3.1. Let $G$ be a finite group and $n$ a positive integer such that

3) $G$ is realizable over $\mathbb{Q}$ by a tamely ramified extension $K / \mathbb{Q}$,

4) $G$ has a subgroup $H$ of index $n$, the intersection of whose conjugates is trivial, such that the set product $M H \neq G$ for every metacyclic subgroup $M$ of $G$.

Then there exists an irreducible polynomial $f(x) \in \mathbb{Q}[x]$ of degree $n$ (with splitting field $K$ ) which is reducible over $\mathbb{Q}_{p}$ for all primes $p$.

Theorem 3.2. For any composite positive integer $n$, there exist irreducible polynomials $f(x) \in \mathbb{Q}[x]$ of degree $n$ which are reducible over $\mathbb{Q}_{p}$ for all primes $p$.

Proof. By Lemma 3.1, it suffices to find $G$ satisfying 3 ) and 4).

Case 1. $n$ squarefree. The same $G$ and $H$ work as in the proof of Theorem 2.2, provided the order $t$ of $q$ mod $m$ is greater than 1 (which can be ensured by taking $q$ to be the smallest prime dividing $n$ ). Indeed, $V$ has $\mathbb{F}_{q}$-dimension $t$ and is irreducible as a $C_{m}$-module, so in this case the only metacyclic subgroups of $G$ are cyclic of order $q$ or a divisor of $m$. Thus condition 4) is satisfied.

Condition 3) can be proved using Saltman's results, [5. Theorem 3.5] to verify that $G$ has a generic Galois extension and then [5, Theorem 5.9] to see that there exists a tame extension realizing $G$.

Case 2. $n$ not squarefree. As in the proof of Theorem 2.2, we first assume $n=q^{2}, q$ a prime. We will construct a Galois extension $K / \mathbb{Q}$ with Galois group $C_{q} \times C_{q}$, with local degree 1 or $q$ at all primes $p$. If $K=\mathbb{Q}(\theta)$ with $f(x)$ the minimal polynomial of $\theta$ over $\mathbb{Q}$, then $f$ has the desired property. As we saw earlier, for any prime unramified in $K$ the decomposition group is cyclic, so the local degree is 1 or $q$ just from the structure of $G$. We need to construct $K$ so that the local degree is $q$ at the ramified primes. For this we use an idea from [3].

Let $\ell$ be a prime congruent to $1 \bmod q$ and let $L_{\ell} \subseteq \mathbb{Q}\left(\mu_{\ell}\right)$ such that $G\left(L_{\ell} / \mathbb{Q}\right) \cong$ $C_{q}$. We seek another prime $r$ such that

5) $r \equiv 1(\bmod q)$ 
6) $r$ splits completely in $L_{\ell}$,

7) $\ell$ splits completely in $L_{r}$.

If $r$ satisfies these conditions, then clearly $K=L_{\ell} L_{r}$ will have the local degree at most $q$ everywhere.

$5)$ is equivalent to the condition that $r$ splits completely in $\mathbb{Q}\left(\mu_{q}\right)$. 7) is equivalent to the condition that the Frobenius automorphism of $\ell$ in $G\left(\mathbb{Q}\left(\mu_{r}\right) / \mathbb{Q}\right)$ fixes $L_{r}$ pointwise, i.e. is a $q$ th power in the cyclic group $G\left(\mathbb{Q}\left(\mu_{r}\right) / \mathbb{Q}\right)$, which is equivalent to $\ell$ being a $q$ th power mod $r$, which in turn means that the polynomial $x^{q}-\ell$ has a root $\bmod r$. Since $\mathbb{F}_{r}$ contains the $q$ th roots of unity, this is equivalent to $x^{q}-\ell$ factoring into linear factors $\bmod r$, which is equivalent to the condition that $r$ splits completely in $\mathbb{Q}\left(\mu_{q}, \sqrt[q]{\ell}\right)$. It follows that conditions 5),6), 7) together are equivalent to the condition that $r$ splits completely in $L_{\ell}\left(\mu_{q}, \sqrt[q]{\ell}\right)$. By Chebotarev's density theorem, such an $r$ exists. This completes the case $n=q^{2}$.

Now assume the general case $n=q^{2} m, q$ prime. Let $K$ be as in the case $n=q^{2}$, and let $L$ be any abelian extension of $\mathbb{Q}$ of degree $m$ such that $K \cap L=\mathbb{Q}$. (For example, choose a prime $p \equiv 1(\bmod m), p \neq \ell, r$, and let $L \subseteq \mathbb{Q}\left(\mu_{p}\right)$ of degree $m$ over $\mathbb{Q}$.) $K L / \mathbb{Q}$ has degree $n$, and the local degree at any prime is at most $q m<n$.

\section{Global fields}

Theorem 3.2 generalizes to arbitrary global fields $F$. If $n$ is prime and $f(x) \in F[x]$ is a separable monic irreducible polynomial, then by Chebotarev's density theorem, which holds over any global field [7, p. 289], there exist primes $\mathfrak{p}$ of $F$ such that $f$ is irreducible over the completion $F_{\mathfrak{p}}$. (Even if $n$ is equal to the characteristic $p$ of $F$, and $f(x)$ is inseparable of degree $p$, i.e. $f(x)=x^{p}-a$, then by [1, Chapter 9 , Theorem 1], if $f(x)$ is irreducible, then $f(x)$ is irreducible over $F_{\mathfrak{p}}$ for infinitely many $\mathfrak{p}$.)

Theorem 4.1. For any composite positive integer $n$, and any global field $F$, there exist irreducible polynomials $f(x) \in F[x]$ of degree $n$ which are reducible over $F_{\mathfrak{p}}$ for all primes $\mathfrak{p}$ of $F$.

Proof. If $F$ is a number field, one can reduce the proof to the case $F=\mathbb{Q}$. Let $f(x) \in \mathbb{Q}[x]$ be irreducible of degree $n$ and reducible over $\mathbb{Q}_{p}$ for all $p$, and suppose its splitting field $K$ satisfies $K \cap F=\mathbb{Q}$. Then $f(x)$ is irreducible over $F$ and is reducible over $F_{\mathfrak{p}}$ for all primes $\mathfrak{p}$ of $F$. It remains to observe that the proof of Theorem 3.2 produces infinitely many $\mathbb{Q}$-linearly disjoint extensions $K$ with the desired properties. We may therefore assume that $F$ a global function field of characteristic $p$.

Let $f(x) \in F[x]$ be monic irreducible of degree $n$, fix a root $\alpha$ in a splitting field $K$, let $G=G(K / F)$, and let $H=G(K / F(\alpha))$. Let $\mathfrak{p}$ be a prime of $F, \mathfrak{P}$ a prime of $K$ dividing $\mathfrak{p}, D=D(\mathfrak{P})$ the decomposition group. Then $f(x)$ is reducible over $F_{\mathfrak{p}}$ if and only if $D$ does not act transitively on the roots of $f(x)$, i.e. the set $D H$ is not equal to $G$. We reduce the proof to the case when $n$ is a product of two primes, not necessarily distinct.

For any positive integer $m$, let $E / F$ be a Galois (e.g. cyclic) extension of degree $m$ such that $K \cap E=F$, with $K$ as above. Assume for all primes $\mathfrak{P}$ of $K$ that $D(\mathfrak{P}) H \neq G$. Let $\hat{K}=K E, \hat{G}=G(K E / F) \cong G \times G(E / F)$. Identify $H$ with $H \times\{1\} \subset \hat{G}$. Then for any prime $\hat{\mathfrak{P}}$ of $\hat{K}, D(\hat{\mathfrak{P}}) H \neq \hat{G}$. This reduces the proof 
of Theorem 4.1 to the case when $n$ is a product of two primes, say $r$ and $s$. We will further reduce the proof to the case that $F$ is a rational function field $\mathbb{F}_{q}(t)$, by constructing the desired $K / \mathbb{F}_{q}(t)$ linearly disjoint over $\mathbb{F}_{q}(t)$ from any $F$ given in advance. Accordingly we now assume $F=\mathbb{F}_{q}(t)$. As we will see, when $n$ is prime to $p$, one can give a proof which is analogous to that of Theorem 3.2.

Case 1. $r=s \neq p$. We use a function field analogue of a construction in [3]. Let $\mathfrak{p}=(h(t))$ be a (finite) prime of $F\left(h(t) \in \mathbb{F}_{q}[t]\right)$ which splits completely in the extension $F^{\prime}$ of $F$ obtained by adjoining all $r$ th roots of all elements of $\mathbb{F}_{q}$ (including the $r$ th roots of unity). Then $C l_{F}(\mathfrak{p})$, the ray class group mod $\mathfrak{p}$, is cyclic (the class group of $F$ is trivial), and has order divisible by $r$. There is a corresponding ray class field extension $R^{\mathfrak{p}} / F$ which is geometric (regular over $\left.\mathbb{F}_{q}\right), G\left(R^{\mathfrak{p}} / F\right) \cong C l_{F}(\mathfrak{p})$, and $\mathfrak{p}$ is the only prime that ramifies in $R^{\mathfrak{p}}$. Let $L^{\mathfrak{p}}$ be the (unique, cyclic) subfield of $R^{\mathfrak{p}}$ of degree $r$ over $F$. We seek $\mathfrak{p}, \mathfrak{q}$ such that $K:=L^{\mathfrak{p}} L^{\mathfrak{q}}$ has the desired property, which is that $\mathfrak{p}$ splits completely in $L^{\mathfrak{q}}$ and $\mathfrak{q}$ splits completely in $L^{\mathfrak{p}}$. Let $\mathfrak{p}$ be as above, which exists e.g. by Chebotarev's density theorem (we don't really need Chebotarev yet since the extension is a constant extension, but presently we will need it). We seek $\mathfrak{q}$ which satisfies the same conditions as does $\mathfrak{p}$, and additionally, $\mathfrak{p}$ splits completely in $L^{\mathfrak{q}}$ and $\mathfrak{q}$ splits completely in $L^{\mathfrak{p}}$. The last condition is an additional Chebotarev condition on $\mathfrak{q}$ which is compatible with the preceding ones, so can be satisfied by Chebotarev's density theorem. The condition $\mathfrak{p}$ splits completely in $L^{\mathfrak{q}}$ is equivalent to the condition that the Frobenius Frob(p) is an $r$ th power in $G\left(R^{\mathfrak{q}} / F\right)$, which is equivalent to $\mathfrak{p}=h(t)$ being an $r$ th power in $C l_{F}(\mathfrak{q})$, which is equivalent to $h(t)$ being an $r$ th power in the multiplicative group of the residue field $\mathbb{F}_{q}[t] / \mathfrak{q}$, which is equivalent to $\mathfrak{q}$ splitting completely in $F(\sqrt[r]{h(t)})$. This is another Chebotarev condition on $\mathfrak{q}$ compatible with the preceding ones. The construction yields infinitely many linearly disjoint extensions $K$, completing the proof of this case.

Case 2. $r \neq s$, both $\neq p$. Here we can argue exactly as in the case $n$ squarefree in the proof of Theorem 3.2, by constructing a tamely ramified $G$-extension using Saltman's results as quoted earlier. This yields infinitely many linearly disjoint extensions.

Case 3. $r=s=p$. We seek a pair of Artin-Schreier extensions $L, M$ defined by $x^{p}-x-g(t), x^{p}-x-h(t)$ respectively, where $g(t), h(t) \in F$, such that $K:=L M$ has local degree 1 or $p$ everywhere. Let $g(t)$ be a polynomial without constant term, i.e. $g(0)=0$. Since the derivative of $x^{p}-x-g(t)$ with respect to $x$ is -1 , the only prime of $F$ that ramifies in $L$ is infinity. Furthermore, the prime corresponding to $t$ splits completely in $L$ since $x^{p}-x-g(t)$ has $p$ distinct roots mod $t$. The automorphism $t \mapsto 1 / t$ interchanges $(t)$ and infinity. Let $h(t)=g(1 / t)$. Then infinity splits completely in $M$ and $(t)$ is the only ramified prime in $M$. It follows that the local degree of $L M$ is 1 or $p$ everywhere, as desired. We get infinitely many linearly disjoint extensions by varying $g(t)$, e.g. by taking $g(t)$ of the form $t^{e}$, with $e$ prime to $p$. The genus of the corresponding curve grows with $e$, or one can show that there are infinitely many such distinct extensions even over the completion of $F$ at infinity.

Case 4. $r=p \neq s$.

Subcase 4.1. $p \nmid s-1$. We can use the group $G$ we used earlier. Let $C_{p}$ act on an irreducible $\mathbb{F}_{s}$-space $V$, so $\operatorname{dim}(V)>1$. Claim that $G:=C_{p} \ltimes V$ cannot be a local Galois group anywhere. Indeed, if it were, the extension would be ramified, 
so the inertia group would be a nontrivial normal subgroup of $G$, hence equal to $V$ or $G$. There is no wild ramification, since $G$ has no normal $p$-subgroup. But then the inertia subgroup must be cyclic, contradicting the fact that $G$ has no cyclic normal subgroup. This proves the claim. It follows that all decomposition groups are proper subgroups of $G$. But if $D$ is a proper subgroup of $G$, then $D H$ cannot equal $G$, as is easily verified (see beginning of the previous section). It remains to show that $G$ is realizable (infinitely often, linearly disjointly) over $F$. Realize $C_{p}$ by an Artin-Schreier extension $L / F$ (there are infinitely many), and consider the embedding problem with kernel $V$. It has a proper solution since $F$ is Hilbertian 4. p. 275].

Subcase 4.2. $p \mid s-1$. Here the roles of $p$ and $s$ need to be interchanged, and we have to worry about wild ramification. (The argument will also cover subcase 4.1.) Let $E / F$ be a cyclic regular extension of degree $s$. Claim there exists an ArtinSchreier extension $L / E$ of degree $p$ such that every prime $\mathfrak{q}$ of $E$ that ramifies in $L$ is split completely over $F$ and all its remaining $s-1$ conjugates over $F$ are unramified in $L$. Indeed, there are infinitely many primes $\mathfrak{p}$ of $F$ that split completely in $E$. Let $\left\{\mathfrak{p}_{i}\right\}$ be a sequence of such primes and let $\mathfrak{q}_{i}$ be a prime of $E$ dividing $\mathfrak{p}_{i}$ for each $i$. Let $h=h_{E}$ be the class number of $E$. Then in the sequence $\mathfrak{q}_{1}, \mathfrak{q}_{1} \mathfrak{q}_{2}, \ldots, \mathfrak{q}_{1} \mathfrak{q}_{2} \cdots \mathfrak{q}_{h+1}$ of ideals of $E$, there must be two which differ by a principal ideal $(f)$; i.e., there exist $i \leq j \in\{1,2, \ldots, h+1\}$ such that $\mathfrak{q}_{i} \cdots \mathfrak{q}_{j}=(f)$ with $f \in E^{*}$. Take $L / E$ to be the Artin-Schreier extension defined by the equation $x^{p}-x=1 / f$. Since the only poles of $1 / f$ are $\mathfrak{q}_{i}, \ldots, \mathfrak{q}_{j}$, the only ramified primes in $L / E$ are also $\mathfrak{q}_{i}, \ldots, \mathfrak{q}_{j}$. Furthermore, since these are simple poles, the extension $L / E$ is nontrivial (of degree $p$ ). This proves the claim. Let $M / F$ be the Galois closure of $L / F$, and set $W=G(M / E)$. By construction, the conjugates of $L$ over $F$ are linearly disjoint over $E$, so $W$ is isomorphic to the group $\operatorname{ring} \mathbb{F}_{p} C_{s}$ as $C_{s}$-modules, identifying $G(E / F)$ with $C_{s}$. There is an irreducible submodule $V$ of $W$ on which $C_{s}$ acts faithfully. The $\mathbb{F}_{p}$-dimension of $V$ is necessarily greater than 1 since $s>p$. Let $K$ be the subfield of $M$ corresponding to the complementary submodule to $V$ in $W$ (by Maschke's theorem). Then $K / F$ is Galois with group $G=C_{s} \ltimes V$. Let $H$ be a subgroup of $V$ of index $p$. We show that $H$ has the desired property, that $D H \neq G$ for any decomposition group $D$. The argument is similar to previous ones. If $D$ is the decomposition group of an unramified prime, then $D$ is cyclic. Since $G$ has no cyclic subgroup of order $s p, D$ has order $p$ or $s$; hence $D H$ cannot equal $G$. At a tamely ramified prime, the inertia group must be of order $s$ and be normal in $D$. So $D$ is either cyclic of order $s$, and $D H \neq G$, or $D$ is of order $s p$ with a normal $p$-Sylow subgroup, which cannot happen because $D$ has no element of order $s p$. Finally, the decomposition group of a wildly ramified prime in $K / F$ must be contained in $V$, since such a prime must divide one of $\mathfrak{q}_{i}, \ldots \mathfrak{q}_{j}$, and $C_{s}$ does not fix any of them. Hence again $D H \neq G$. Finally, there are infinitely many linearly disjoint extensions $E / F$, hence infinitely many linearly disjoint extensions $K / F$. The proof is complete.

\section{REFERENCES}

[1] E. Artin and J. Tate, Class Field Theory, Harvard University, Cambridge, 1961. MR1043169 (91b:11129)

[2] R. Brandl, Integer polynomials that are reducible modulo all primes, Amer. Math. Monthly 93 (1986), 286-288. MR0835298 (87f:12007) 
[3] H. Kisilevsky and J. Sonn, On the n-torsion subgroup of the Brauer group of a number field, J. Th. Nombres de Bordeaux 15 (2003), 199-204. MR.2019011 (2004j:11142)

[4] G. Malle and B.H. Matzat, Inverse Galois Theory, Springer-Verlag, Berlin, 1999. MR 1711577 (2000k:12004)

[5] David Saltman, Generic Galois extensions and problems in field theory, Adv. Math. 43 (1982), 250-283. MR0648801 (84a:13007)

[6] B. L. Van der Waerden, Die Seltenheit der Gleichungen mit Affekt, Math. Ann. 109 (1934), 13-16.

[7] A. Weil, Basic Number Theory, third ed., Springer-Verlag, Berlin, 1974. MR0427267(55:302)

Department of Mathematics, University of Southern California, Los Angeles, CalIFORNIA 90089-2532

E-mail address: guralnic@usc.edu

Department of Mathematics, University of California at los Angeles, Los Angeles, CALIFORNIA 90024

E-mail address: mms@math.ucla.edu

Department of Mathematics, Technion, 32000 Haifa, IsRael

E-mail address: sonn@math.technion.ac.il 